\title{
mHealth mood monitoring for people with COPD
}

Maxine Whelan, Carmelo Velardo, Heather Rutter, Lionel Tarassenko, Andrew Farmer

European Respiratory Journal 2019 54: PA2236; DOI: 10.1183/13993003.congress-2019.PA2236

\begin{abstract}
Background: Co-morbid anxiety and depression can add to the complexity of chronic obstructive pulmonary disease (COPD). While digital health technologies for people with COPD support monitoring of vital signs (including oxygen saturation and pulse), mood is not routinely monitored even though it could support self-management.

Aim: To report adherence to mood self-monitoring and levels of anxiety and depression for people with COPD.

Methods: The sElf-management anD support proGrammE (EDGE) was a 12-month randomised controlled trial involving people with COPD. Participants were asked to complete an anxiety (General Anxiety Disorder, GAD) and depression questionnaire (Patient Health Questionnaire, PHQ) every 4 weeks via a tablet computer. Health status (EuroQol 5 Dimensions, EQ5D), respiratory impairment (St George's Respiratory Questionnaire-COPD, SGRQ-C) and smoking status were recorded. Answering $\geq 10$ of 12 months was adherent and PHQ and GAD scores of $\geq 5$ positive. Results: Of 106 (99\%) participants entering mood data, 81 participants were adherent. There were more ex-smokers in the adherent participant pool (n=71 of 81) compared with the non-adherent participants $(n=13$ of 25); $\mathrm{p}<0.001$. Participants with positive PHQ and GAD scores $(n=56)$ compared with those without $(n=22)$ had EQ5D scores of mean \pm SD $0.6 \pm 0.2$ and $0.7 \pm 0.2$, respectively, and SGRQ-C scores of $62 \pm 19$ and $50 \pm 16$, respectively; both $\mathrm{p}<0.05$.

Conclusions: People with COPD can self-monitor mood over 12 months. With $>50 \%$ of participants suggesting an elevated risk of both anxiety and depression (associated with a lower health status and higher respiratory impairment), the opportunity to regularly monitor mood in this patient population could offer useful information for comorbidity management.
\end{abstract}

